\title{
Stability and invariance results for a class of non-monotone set-valued Lur'e dynamical systems
}

\author{
Samir Adly* and Ba Khiet Le \\ XLIM UMR-CNRS 7252, Université de Limoges, 87060 Limoges, France
}

\begin{abstract}
In this paper, we analyse the well-posedness, stability and invariance results for a class of non-monotone set-valued Lur'e dynamical system which has been widely studied in control and applied mathematics. Many recent researches deal with the case when the set-valued part is the sub-differential of some proper, convex, lower semicontinuous function in order to use the nice properties of maximally monotone operators. But in practice, particularly in electronics, there are some devices such as diac, silicon controller rectifier (SCR) ... that their voltagecurrent characteristics are not monotone but only locally hypo-monotone. This fact motivates us to write the paper which is organized as follows: firstly, the existence and uniqueness of solutions are proved by using Filippov's method and local hypo-monotonicity; then, the stability analysis and generalized LaSalle's invariance principle are presented. The theoretical results are supported by numerical simulations for some examples in electronics. Our methology is based on non-smooth and variational analysis.
\end{abstract}

Keywords: well-posedness; stability analysis; LaSalle's invariance principle; hypo-monotone; Filippov's solutions; electronic devices

\section{Introduction}

The general Lur'e systems are the systems which have a negative feedback interconnection of an ordinary differential equation $\dot{x}(t)=f(x(t), p(t))$ where $p$ is one of the two slack variables, with the second one $q=g(x, p)$ and satisfy the inclusion condition $p \in \Phi(t, q)$. There is a remark that other mathematical models used to study non-smooth dynamical systems (relay systems, evolution variational inequalities, projected dynamical systems, complementarity systems etc.) can be also recast into Lur'e systems with a set-valued feedback part.[1-3]

We are interested in the Lur'e systems which are (possibly nonlinear) time-invariant dynamical systems with static set-valued feedback. Usually, the function $g$ has the form: $g(x, p)=\mathcal{C} x+D p$. The case $D=0$ appears in many applications in electronics particularly, while the case $D \neq 0$ is more general but creates some difficulties when one wants to study the possibly set-valued operator $\left(-D+\Phi^{-1}\right)^{-1}(\mathcal{C} \circ \cdot)$. In [4], Brogliato

*Corresponding author. Email: samir.adly@unilim.fr 


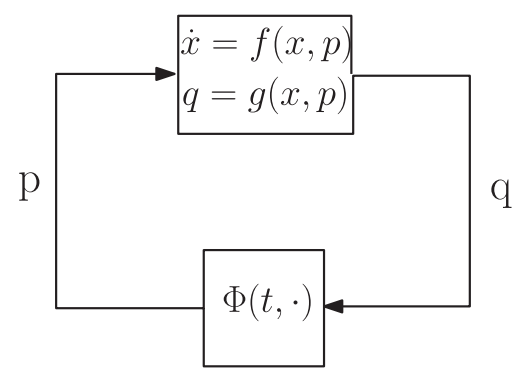

Figure 1. Lur'e systems.

and Goeleven overcome these obstacles by assuming that $\Phi$ is the sub-differential of some proper, convex, lower semi-continuous function to enjoy the nice properties of Fenchel transform and maximally monotone operators. However, the case of $D=0$ is still interesting since it includes applications in practice such as the electrical circuits with some devices where their voltage-current characteristics are not monotone. There are certainly many results for the null matrix $D$, but very few ones for the case of non-monotone set-valued parts. This is the aim of our work, which is hoped to fill some gaps in the study literature of Lur'e systems.

In this paper, we reformulate a class of Lur'e systems into the first-order differential inclusion form where the set-valued right-hand side is upper semi-continuous with non-empty, convex, compact values to obtain the existence of a solution. Then, local hypo-monotonicity is assumed to ensure the uniqueness result. Next, we give a stability analysis and extend LaSalle's invariance principle to such systems. Finally, some illustrative examples in electronics are presented with numerical simulations. The methodology used in this paper is based on non-smooth and variational analysis[5,13-16]

\section{Notation and mathematical preliminaries}

Denote by $\langle\cdot, \cdot\rangle,\|\cdot\|$ the scalar product and the corresponding norm (the euclidean norm) in $\mathbb{R}^{n}, \mathbb{B}_{\varepsilon}$ the closed ball of radius $\varepsilon$ centred at 0 and $\mathbb{B}_{\varepsilon}(a)$ the closed ball of radius $\varepsilon$ centered at $a$. The dimension of the ball can be implied by the context without any confusion. Let $F: \mathbb{R}^{n} \rightrightarrows \mathbb{R}^{n}$ be a set-valued function.

Definition 1 One says that $F$ is upper semi-continuous at $x_{0} \in \mathbb{R}^{n}$ if for any open neighbourhood $\mathcal{N}$ containing $F\left(x_{0}\right)$, there exists an open neighbourhood $\mathcal{M}$ of $x_{0}$ such that $F(\mathcal{M}) \subset \mathcal{N}$. If $F$ is upper semi-continuous at every $x_{0} \in \mathbb{R}^{n}$, then it is called upper semi-continuous on $\mathbb{R}^{n}$.

Remark 1 If $F$ is a single-valued function on $\mathbb{R}$, this definition is equivalent to continuity of $F$, which is different from the known definition of "upper semi-continuous" for singlevalued functions. Hence, the mapping $F$ is also called "outer semi-continuous" by some authors instead of "upper semi-continuous". In this paper, we are interested in the case of set-valued function, so we can keep the term "upper semi-continuous" conventionally without vagueness.

Proposition 1 ([5]) Let $F_{1}: \mathbb{R}^{n} \rightrightarrows \mathbb{R}^{m}$ and $F_{2}: \mathbb{R}^{m} \rightrightarrows \mathbb{R}^{q}$ be two set-valued functions. Define $F_{2} \circ F_{1}: \mathbb{R}^{n} \rightrightarrows \mathbb{R}^{q}$ by: 


$$
\left(F_{2} \circ F_{1}\right)(x)=\bigcup_{y \in F_{1}(x)} F_{2}(y)
$$

If $F_{1}$ and $F_{2}$ are upper semi-continuous, then $F_{2} \circ F_{1}$ is upper semi-continuous.

Definition $2 \quad F: \mathbb{R}^{n} \rightrightarrows \mathbb{R}^{n}$ is called hypo-monotone provided that there exists a real $k>0$ such that for all $x_{1}, x_{2} \in \mathbb{R}^{n}$, we have:

$$
\left\langle y_{1}-y_{2}, x_{1}-x_{2}\right\rangle \geq-k\left\|x_{1}-x_{2}\right\|^{2}, \quad \forall y_{1} \in F\left(x_{1}\right), y_{2} \in F\left(x_{2}\right),
$$

or we can write:

$$
\left\langle F\left(x_{1}\right)-F\left(x_{2}\right), x_{1}-x_{2}\right\rangle \geq-k\left\|x_{1}-x_{2}\right\|^{2} .
$$

The map $F(\cdot)$ is called locally hypo-monotone if for each $x_{0} \in X, F(\cdot)$ is hypo-monotone in a neighbourhood of $x_{0}$.

Lemma 1 If $F: \mathbb{R}^{n} \rightrightarrows \mathbb{R}^{n}$ is hypo-monotone (resp. locally hypo-monotone), then $C^{T}$ 。 $F \circ C: \mathbb{R}^{m} \rightrightarrows \mathbb{R}^{m}$ is also hypo-monotone (resp. locally hypo-monotone) for any matrix $C \in \mathbb{R}^{n \times m}$.

Proof It can be implied directly by the following estimation:

$$
\begin{aligned}
\left\langle C^{T} F(C x)-C^{T} F(C y), x-y\right\rangle & =\langle F(C x)-F(C y), C x-C y\rangle \geq-k\|C x-C y\|^{2} \\
& \geq-k\|C\|_{i}^{2}\|x-y\|^{2},
\end{aligned}
$$

for all $x, y \in \mathbb{R}^{m}$ where $\|\cdot\|_{i}$ is the induced matrix norm.

Definition $3 \quad F: \mathbb{R}^{n} \rightrightarrows \mathbb{R}^{n}$ is said to satisfy the linear growth condition if there exists a positive real number $c$ such that for all $x \in \mathbb{R}^{n}$ :

$$
\|w\| \leq c(1+\|x\|), \quad \forall w \in F(x) .
$$

Let $x_{0}$ be some given element of $\mathbb{R}^{n}$ and $F: \mathbb{R}^{n} \rightrightarrows \mathbb{R}^{n}$ be a set-valued function. We consider the differential inclusion problem: find an absolutely continuous function $x\left(. ; x_{0}\right)$ such that:

$$
\left\{\begin{array}{l}
x^{\prime}(t) \in F(x(t)), \text { a.e. } t \in[0,+\infty), \\
x(0)=x_{0} .
\end{array}\right.
$$

The following theorem is a fundamental result in the Theory of Filippov (see [6]).

Theorem 1 Let $F: \mathbb{R}^{n} \rightrightarrows \mathbb{R}^{n}$ be an upper semi-continuous set-valued function with non-empty, compact and convex values satisfying the linear growth condition (1). Then, for all $x_{0} \in \mathbb{R}^{n}$, there exists an absolutely continuous function $x\left(. ; x_{0}\right)$ defined on $[0,+\infty)$ which is a solution of (2).

\section{Non-smooth Lur'e dynamical system}

Let $A: \mathbb{R}^{n} \rightarrow \mathbb{R}^{n}$ be a (possibly) non-linear operator, $B \in \mathbb{R}^{n \times m}, \mathcal{C} \in \mathbb{R}^{m \times n}$ be given matrices; $\mathcal{F}_{i}: \mathbb{R} \rightrightarrows \mathbb{R}(i=1,2, \ldots, m)$ be given upper semi-continuous mappings with 
non-empty, convex, compact values; $p=\left(p_{1}, \ldots, p_{m}\right)^{T}, q=\left(q_{1}, \ldots, q_{m}\right)^{T}: \mathbb{R}^{+} \rightarrow \mathbb{R}^{m}$ be two unknown mappings. For $x_{0} \in \mathbb{R}^{n}$, we consider the following problem: find an absolutely continuous function $x(\cdot)$ defined on $[0,+\infty)$ such that:

$$
\left\{\begin{array}{l}
x^{\prime}(t)=A(x(t))+B p(t) \text { a.e. } t \in[0,+\infty) \\
q(t)=\mathcal{C} x(t) \\
p_{i}(t) \in \mathcal{F}_{i}\left(q_{i}(t)\right), i=1,2, \ldots, m, \forall t \geq 0 \\
x(0)=x_{0}
\end{array}\right.
$$

Particularly in electrical circuits, the inclusion $p_{i}(t) \in \mathcal{F}_{i}\left(q_{i}(t)\right)$ may represent the voltagecurrent characteristics of some electronic devices. Note that, $\mathcal{F}_{i}$ here maybe a non-monotone operator for some $i \in\{1,2, \ldots, m\}$. In practice, we are interested in the state variable $x(\cdot)$. However, in our system, $q(\cdot)$ can be computed uniquely in the term of $x(\cdot)$, so $q(\cdot)$ is also absolutely continuous. The mapping $p(\cdot)$ may be found uniquely by $x(\cdot)$ almost everywhere. Its properties depend on the regularity of the set-valued mapping $\mathcal{F}_{i}$. For example, under some mild conditions of $\mathcal{F}_{i}$ for $i=1,2, \ldots, m$ then $p(\cdot)$ is Lipschitz continuous ([4]).

Let $F: \mathbb{R}^{m} \rightrightarrows \mathbb{R}^{m}, q=\left(q_{1}, \ldots, q_{m}\right)^{T} \rightarrow F(q)$ defined by:

$$
F(q)=\left(\mathcal{F}_{1}\left(q_{1}\right), . ., \mathcal{F}_{m}\left(q_{m}\right)\right)^{T} .
$$

It is easy to see that (3) can be rewritten as:

$$
\left\{\begin{array}{l}
x^{\prime}(t)=A(x(t))+B p(t) \text { a.e. } t \in[0,+\infty) \\
q(t)=\mathcal{C} x(t) \\
p(t) \in F(q(t)), \text { for all } \mathrm{t} \geq 0 \\
x(0)=x_{0}
\end{array}\right.
$$

We will show in Lemma 2 that $F$ is also an upper semi-continuous mapping with non-empty, convex and compact values.

Lemma 2 If $\mathcal{F}_{i}: \mathbb{R} \rightrightarrows \mathbb{R}$ is an upper semi-continuous mapping with non-empty, convex and compact values for each $i=1, \ldots, m$ then $F: \mathbb{R}^{m} \rightrightarrows \mathbb{R}^{m}$ defined in (4) is also an upper semi-continuous mapping with non-empty, convex and compact values.

Proof It is clear that $F$ has non-empty, convex and compact values. It remains to prove that $F$ is upper semi-continuous. Given $\epsilon>0$ and $x=\left(x_{1}, \ldots, x_{m}\right)^{T}$. Then, we can choose $\bar{\epsilon}=$ $\epsilon / \sqrt{m}>0$ such that $\left(\mathbb{B}_{\bar{\epsilon}}\left(\mathcal{F}_{1}\left(x_{1}\right)\right), \ldots, \mathbb{B}_{\bar{\epsilon}}\left(\mathcal{F}_{m}\left(x_{m}\right)\right)\right)^{T} \subset \mathbb{B}_{\epsilon}\left(\left(\mathcal{F}_{1}\left(x_{1}\right), \ldots, \mathcal{F}_{m}\left(x_{m}\right)\right)^{T}\right)=$ $\mathbb{B}_{\epsilon}(F(x))$. Since for each $i=1, \ldots, m$, the mapping $\mathcal{F}_{i}$ is upper semi-continuous, there exists $\bar{\delta}>0$ such that $\mathcal{F}_{i}\left(\mathbb{B}_{\bar{\delta}}\left(x_{i}\right)\right) \subset \mathbb{B}_{\bar{\epsilon}}\left(\mathcal{F}_{i}\left(x_{i}\right)\right)$. Finally, we take $\delta=\bar{\delta}$ then $\mathbb{B}_{\delta}(x) \subset$ $\left(\mathbb{B}_{\bar{\delta}}\left(x_{1}\right), \ldots, \mathbb{B}_{\bar{\delta}}\left(x_{n}\right)\right)^{T}$ and hence $F\left(\mathbb{B}_{\delta}(x)\right) \subset\left(\mathcal{F}_{1}\left(\mathbb{B}_{\bar{\delta}}\left(x_{1}\right)\right), \ldots, \mathcal{F}_{m}\left(\mathbb{B}_{\bar{\delta}}\left(x_{m}\right)\right)\right)^{T}$. So, we have found a $\delta$ such that $F\left(\mathbb{B}_{\delta}(x)\right) \subset \mathbb{B}_{\epsilon}(F(x))$. Hence, $F$ is upper semi-continuous. 
Remark 2 If $\mathcal{F}_{i}$ is hypo-monotone (resp. locally hypo-monotone) for each $i=1, \ldots, m$, then $F$ defined in (4) is also hypo-monotone (resp. locally hypo-monotone). Indeed, for all $x=\left(x_{1}, \ldots, x_{n}\right)^{T}, y=\left(y_{1}, \ldots, y_{n}\right)^{T} \in \mathbb{R}^{n}$, we have:

$$
\begin{aligned}
\langle F(x)-F(y), x-y\rangle & =\left(\mathcal{F}_{1}\left(x_{1}\right)-\mathcal{F}_{1}\left(y_{1}\right)\right)\left(x_{1}-y_{1}\right)+\ldots+\left(\mathcal{F}_{n}\left(x_{n}\right)-\mathcal{F}_{n}\left(y_{n}\right)\right)\left(x_{n}-y_{n}\right) \\
& \geq-k_{1}\left|x_{1}-y_{1}\right|^{2}-\ldots-k_{n}\left|x_{n}-y_{n}\right|^{2} \geq-k\|x-y\|^{2} .
\end{aligned}
$$

where $k=\max \left\{k_{1}, \ldots, k_{n}\right\}$.

\section{Existence and uniqueness}

In this section, we recast the Lur'e system into the Filippov case to obtain the existence of a solution. Each solution can be extended globally by using the linear growth condition. Then, local hypo-monotonicity of the right-hand side is supposed to have the uniqueness of the solutions.

Indeed, the system (3) can be reduced to the first-order differential inclusion:

$$
x^{\prime}(t) \in \mathcal{Q}(x(t)):=A(x(t))+B F(\mathcal{C} x(t)) \quad \text { a.e. } t \in[0,+\infty) .
$$

Theorem 2 Suppose that $A$ is $k$-lipschitz and there exists a positive constant $c_{F}$ such that:

$$
\|w\| \leq c_{F}(1+\|y\|), \quad \forall w \in F(y), \forall y \in \mathbb{R}^{m} .
$$

Then, for every $x_{0} \in \mathbb{R}^{n}$, there exists a global solution of (6).

Proof It is easy to check that $A$ satisfies the linear growth condition. Indeed, we have: $\|A(x)\| \leq\|A(0)\|+k\|x\|$ for all $x \in \mathbb{R}^{n}$. Note that $A$ is continuous and from (7), we imply that, $\mathcal{Q}$ is upper semi-continuous mapping with non-empty, convex, compact values and satisfies the linear growth condition. Then, for all initial condition $x_{0} \in \mathbb{R}^{n}$, there exists an absolutely continuous function $x\left(\cdot ; x_{0}\right)$ satisfying:

$$
x(0)=x_{0},
$$

and

$$
x^{\prime}(t) \in \mathcal{Q}(x(t)) \quad \text { a.e. } t \in[0,+\infty) .
$$

Therefore, we obtain the existence of a solution of problem (6).

Theorem 3 If $-F$ is locally hypo-monotone and there exists an invertible matrix $R \in$ $\mathbb{R}^{n \times n}$ such that:

$$
\mathcal{C}=B^{T} R^{T} R
$$

then (6) has at most one solution.

Proof The system (6) is equivalent to:

$$
R \dot{x} \in R A\left(R^{-1} R x\right)+R B F\left(B^{T} R^{T} R x\right) .
$$

Setting $z=R x$, we get:

$$
\dot{z} \in R A\left(R^{-1} z\right)+R B F\left(B^{T} R^{T} z\right) .
$$


Note that $(R B)^{T}=B^{T} R^{T}$, from Remark 2 and Lemma 1 we obtain $-R B \circ F \circ B^{T} R^{T}$ is locally hypo-monotone. The mapping $R \circ A \circ R^{-1}$ is Lipschitz. Hence, if for all $z \in \mathbb{R}^{n}$ we set:

$$
\phi(z)=R A\left(R^{-1} z\right)+R B F\left(B^{T} R^{T} z\right),
$$

then $-\phi: \mathbb{R}^{n} \rightrightarrows \mathbb{R}^{n}$ is locally hypo-monotone. Given arbitrary $T>0$. Suppose that $x_{1}(\cdot), x_{2}(\cdot)$ are two solutions of $(6)$ on $[0, T]$ with the same initial conditions $x_{1}(0)=$ $x_{2}(0)=x_{0}$. Then $z_{i}(\cdot):=R x_{i}(\cdot), i=1,2$ are two solutions of (8) satisfying $z_{1}(0)=$ $z_{2}(0)=z_{0}:=R x_{0}$. Since $-\phi$ is locally hypo-monotone, there exist $\epsilon>0$ and $k>0$ such that $-\phi$ is hypo-monotone in $\mathbb{B}_{\epsilon}\left(z_{0}\right)$ with constant $k$. Note that, $z_{1}(\cdot)$ and $z_{2}(\cdot)$ are absolutely continuous functions. Hence, we can find a positive $T_{0} \leq T$ such that $z_{1}(t), z_{2}(t) \in \mathbb{B}_{\epsilon}\left(z_{0}\right)$ for all $t \in\left[0, T_{0}\right]$. From the definition of hypo-monotonicity, we imply that:

$$
\left\langle-\dot{z}_{1}(t)+\dot{z}_{2}(t), z_{1}(t)-z_{2}(t)\right\rangle \geq-k\left\|z_{1}(t)-z_{2}(t)\right\|^{2},
$$

or, equivalently:

$$
\left\langle\dot{z}_{1}(t)-\dot{z}_{2}(t), z_{1}(t)-z_{2}(t)\right\rangle \leq k\left\|z_{1}(t)-z_{2}(t)\right\|^{2},
$$

which means:

$$
\frac{1}{2} \frac{d}{d t}\left\|z_{1}(t)-z_{2}(t)\right\|^{2} \leq k\left\|z_{1}(t)-z_{2}(t)\right\|^{2} .
$$

By Gronwall's inequality, we have $\left\|z_{1}(t)-z_{2}(t)\right\|^{2} \leq 0$ for all $t \in\left[0, T_{0}\right]$, i.e. $z_{1}(t) \equiv z_{2}(t)$ on $t \in\left[0, T_{0}\right]$. We assume that there exists $t_{0} \in[0, T]$ such that $z_{1}\left(t_{0}\right) \neq z_{2}\left(t_{0}\right)$. Let:

$$
\mathcal{E}=\left\{t \in\left[0, t_{0}\right]: z_{1}(t) \neq z_{2}(t)\right\}
$$

Since $t_{0} \in \mathcal{E}$ and $\mathcal{E}$ is bounded from below, there exists $c=\inf \mathcal{E}$ where $c \in\left(0, t_{0}\right]$ and $z_{1}(t)=z_{2}(t)$ for all $t \in[0, c)$. Due to the continuity of $z_{1}(\cdot), z_{2}(\cdot)$, we obtain $z_{1}(c)=z_{2}(c)$ which means that $c<t_{0}$. Using the same argument as above, we can find a neighborhood of $c$ on which $z_{1}(\cdot) \equiv z_{2}(\cdot)$, a contradiction with the definition of $c$. Therefore, we have $z_{1}(\cdot) \equiv z_{2}(\cdot)$ on $[0, T]$ which implies that $x_{1}(\cdot) \equiv x_{2}(\cdot)$ on $[0, T]$. Since $T>0$ is arbitrary, we have proved the result.

\section{Stability and invariance theorems}

In the following section, we give some results about the stability of equilibria and a generalized version of Lasalle's invariance principle is presented. We begin by introducing some basic definitions in the Lyapunov's Theory. Let $x_{0} \in \mathbb{R}^{n}$ and $x\left(t ; x_{0}\right)$ be a solution of (6).

Definition 4 The equilibrium point $x=0$ is said to be stable if

$$
\forall \varepsilon>0, \exists \delta(\varepsilon)>0 \text { such that for all } x_{0} \in \mathbb{B}_{\delta(\varepsilon)} \Rightarrow\left\|x\left(t ; x_{0}\right)\right\| \leq \varepsilon, \forall t \geq 0 .
$$

Definition 5 The equilibrium point $x=0$ is said to be attractive if

$$
\forall \varepsilon>0, \exists \delta(\varepsilon)>0 \text { such that for all } x_{0} \in \mathbb{B}_{\delta(\varepsilon)} \Rightarrow \lim _{t \rightarrow \infty}\left\|x\left(t ; x_{0}\right)\right\|=0 .
$$

If this is true for all $x_{0} \in \mathbb{R}^{n}$, then $x=0$ is said globally attractive. 
Definition 6 If the trivial equilibrium point is stable and attractive, it is called asymptotic stable; if it is stable and globally attractive, it is called globally asymptotic stable.

Let $\mathcal{S}\left(x_{0}\right)$ be the set of solutions and $\mathcal{W}$ be the set of stationary solutions of (6):

$$
\mathcal{W}=\left\{\bar{x} \in \mathbb{R}^{n}: 0 \in \mathcal{Q}(\bar{x})\right\} .
$$

Let us denote the set-valued orbital derivative of a continuously differentiable function $V: \overline{\mathbb{B}}_{\sigma} \subset \mathbb{R}^{n} \rightarrow \mathbb{R}$ (for some $\sigma>0$ ) with respect to the differential inclusion (6):

$$
\dot{V}(x)=\left\{p \in \mathbb{R}: \exists \omega \in \mathcal{Q}(x) \text { such that } p=\left\langle V^{\prime}(x), \omega\right\rangle\right\} .
$$

The upper and lower orbital derivatives of $V$ are sequently defined by:

$$
\dot{V}^{*}(x)=\max _{\omega \in \mathcal{Q}(x)}\left\langle V^{\prime}(x), \omega\right\rangle, \quad \dot{V}_{*}(x)=\min _{\omega \in \mathcal{Q}(x)}\left\langle V^{\prime}(x), \omega\right\rangle .
$$

Remark 3

(1) Since $\mathcal{Q}$ has non-empty, convex and compact values, we have $\dot{V}(x)$ is a nonempty, convex compact subset in $\mathbb{R}$. Therefore, $\dot{V}(x)$ is of the following form: $\dot{V}(x)=\left[\dot{V}_{*}(x), \dot{V}^{*}(x)\right]$. Note that the orbital derivative of more general Lyapunov functions $V$ has been also studied ( for example, see [7]).

(2) If $x(t):=x\left(t ; x_{0}\right)$ is a solution of (6) then:

$$
\frac{d}{d t} V(x(t)) \in \dot{V}(x(t)) \text { a.e. } t \geq t_{0} .
$$

(3) Let $\bar{x} \in \mathcal{W}$, i.e. $0 \in \mathcal{Q}(\bar{x})$. From (10), it is easy to check that $0 \in \dot{V}(\bar{x})$. It means that $\mathcal{W} \subset \mathcal{Z}=\left\{y \in \mathbb{R}^{n}: 0 \in \dot{V}(y)\right\}$. This remark may be used in the next section, when we analyze the asymptotic property of the system by using extended LaSalle's invariance principle.

Definition 7 Let $V: \bar{B}_{\sigma} \subset \mathbb{R}^{n} \rightarrow \mathbb{R}$ be a continuous function such that $V(0)=0$. We say that $V$ is positive definite if $V(x)>0$ for all $x \in \overline{\mathbb{B}}_{\sigma} \backslash\{0\}$.

Definition 8 A Lyaponov function for (6) is a positive definite continuously differentiable function $V: \overline{\mathbb{B}}_{\sigma} \subset \mathbb{R}^{n} \rightarrow \mathbb{R}$ such that $\dot{V}^{*}(x) \leq 0$ for all $x \in \overline{\mathbb{B}}_{\sigma}$.

Assumption $1 \quad 0 \in \mathcal{Q}(0)$, which means that $0 \in \mathcal{W}$.

Remark 4 Let $x^{*}$ be an equilibrium of (6), i.e. $0 \in \mathcal{Q}\left(x^{*}\right)$. If we set $y(\cdot)=x(\cdot)-x^{*}$, then the differential inclusion (6) becomes:

$$
\dot{y}(t) \in \mathcal{Q}_{x^{*}}(y(t)):=\mathcal{Q}\left(y(t)+x^{*}\right) \text { a.e. } t \in[0,+\infty) .
$$

Note that the function $\mathcal{Q}_{x^{*}}(\cdot)$ possesses the same desired properties as $\mathcal{Q}(\cdot)$ has. Furthermore, $0 \in \mathcal{Q}_{x^{*}}(0)$, i.e. the trivial solution is an equilibrium of the new differential inclusion (11). Therefore, it makes sense to propose Assumption 1 as well as to study the stability properties of the origin. 
THEOREM 4 Let the assumption of Theorem 2 and Assumption 1. If there exists a Lyapunov function $V$ for problem (6), then the trivial solution is stable.

Proof Since $V: \overline{\mathbb{B}}_{\sigma} \rightarrow \mathbb{R}$ is positive definite continuously differentiable function, there exist a strictly increasing function $\alpha(\cdot) \in C\left(\mathbb{R}^{+} ; \mathbb{R}\right)$ with $\alpha(0)=0$ and a positive real number, still denoted by $\sigma$ such that:

$$
V(x) \geq \alpha(\|x\|) \text { for all } x \in \overline{\mathbb{B}}_{\sigma} .
$$

Without loss of generality, let $0<\varepsilon<\sigma$ and let $c=\alpha(\varepsilon)$. Since $V$ is positive definite, there exists a $\eta>0$ such that $\mathbb{B}_{\eta} \subset \Omega_{c}^{\circ}=\left\{x \in \mathbb{R}^{n}: V(x)<c\right\}$. Let $\delta=\min \{\varepsilon, \eta\}$. Take $x_{0} \in \mathbb{B}_{\delta}$ and $x\left(t ; x_{0}\right)$ is a solution of (6) satisfying the initial condition $x(0)=x_{0}$. Suppose that there exists $t_{1} \geq 0$ such that $\left\|x\left(t_{1} ; x_{0}\right)\right\| \geq \varepsilon$. Since $x\left(\cdot ; x_{0}\right)$ is continuous, we may find some $t^{*}$ satisfying: $\left\|x\left(t^{*} ; x_{0}\right)\right\|=\varepsilon$. Then,

$$
V\left(x\left(t^{*} ; x_{0}\right)\right) \geq \alpha\left(\left\|x\left(t^{*} ; x_{0}\right)\right\|\right)=\alpha(\varepsilon) .
$$

On the other hand, $V$ is decreasing along the trajectory on the time interval $\left[0, t^{*}\right]$ due to Remark 3.2 and the fact that $\dot{V}^{*}(x) \leq 0$ for all $x \in \overline{\mathbb{B}}_{\sigma}$. Hence, we have:

$$
V\left(x\left(t^{*} ; x_{0}\right)\right) \leq V\left(x_{0}\right)<c=\alpha(\varepsilon) .
$$

Our proof is finished by the contradiction.

THEOREM 5 Let the assumption of Theorem 2 and Assumption 1. If there exists a Lyapunov function $V$ for problem (6) such that $\dot{V}^{*}(x) \leq-\lambda V(x)$ for all $x \in \overline{\mathbb{B}}_{\sigma}$. Then the trivial solution is asymptotic stable.

Proof By the Theorem 4, the trivial solution is stable. Therefore, there exists $\delta>0$ such that for all $x_{0} \in \mathbb{R}^{n}$ and $\left\|x_{0}\right\| \leq \delta$, we have $x\left(t ; x_{0}\right) \in \overline{\mathbb{B}}_{\sigma}$ for all $t \geq 0$. On the other hand, we have: $\frac{d}{d t} V(x(t)) \in \dot{V}(x(t))$ a.e. $t \geq 0$ and $\dot{V}^{*}(x) \leq-\lambda V(x)$ for all $x \in \overline{\mathbb{B}}_{\sigma}$. Hence, we have:

$$
\frac{d}{d t} V(x(t)) \leq-\lambda V(x(t)), \quad \text { a.e. } t \geq 0 .
$$

Using simple integration, we obtain:

$$
V(x(t)) \leq V\left(x_{0}\right) e^{-\lambda t}, \quad t \geq 0 .
$$

Therefore:

$$
0 \leq \alpha(\|x(t)\|) \leq V\left(x_{0}\right) e^{-\lambda t}, \quad t \geq 0 .
$$

Since $\alpha(\cdot)$ is strictly increasing, we must have:

$$
\limsup _{t \rightarrow+\infty}\|x(t)\|=0 .
$$

Therefore:

$$
\lim _{t \rightarrow+\infty}\|x(t)\|=0 .
$$


Remark 5 If we have $\dot{V}^{*}(x) \leq-\lambda V(x)$ for all $x \in \mathbb{R}^{n}$, then the trivial solution is globally asymptotic stable. The proof is similar to the one in Theorem 4.

In the next part of this section, we will generalize the LaSalle's invariance principle to prove the asymptotic stability of the trivial solution. Firstly, we recall some definitions and properties. Let $x_{0} \in \mathbb{R}^{n}$ and $x\left(t ; x_{0}\right)$ be a solution of (6), denote the orbit of $x$ by:

$$
\gamma(x)=\left\{x\left(t ; x_{0}\right): t \geq 0\right\} \subset \mathbb{R}^{n},
$$

and the limit set of $x$ by:

$$
\Lambda(x)=\left\{p \in \mathbb{R}^{n}: \exists\left\{t_{i}\right\}, t_{i} \rightarrow+\infty \text { as } i \rightarrow \infty \text { and } x\left(t_{i} ; x_{0}\right) \rightarrow p\right\} .
$$

A set $S \subset \mathbb{R}^{n}$ is said weakly invariant if and only if for $x_{0} \in S$, there exists a solution of (6) starting at $x_{0}$ contained in $S$. It is said invariant if and only if for $x_{0} \in S$, all solutions of (6) starting at $x_{0}$ are contained in $S$.

\section{Remark 6}

(i) If $\gamma(x)$ is bounded, then $\Lambda(x) \neq \emptyset$ and

$$
\lim _{t \rightarrow \infty} d\left(x\left(t ; x_{0}\right), \Lambda(x)\right)=0 .
$$

If the right-hand side of (6) is upper semi-continuous with non-empty, convex, compact values, we have a result that the limit set $\Lambda(x)$ is weakly invariant $([8,11,12]$ or [6], p. 129).

(ii) The set of stationary solutions $\mathcal{W}$ is weakly invariant. Indeed, if $x_{0} \in \mathcal{W}$, then the solution $x\left(t ; x_{0}\right)=x_{0}, t \geq t_{0}$, is contained in $\mathcal{W}$. From Remark $3.3, \mathcal{W}$ is a weakly invariant subset of $\mathcal{Z}=\left\{y \in \mathbb{R}^{n}: 0 \in \dot{V}(y)\right\}$.

(iii) If for each $x_{0} \in \mathbb{R}^{n}$, the set of solutions $\mathcal{S}\left(x_{0}\right)$ has a unique element, then a weakly invariant set is also invariant. Hence, in the following part, we focus on the case of non-unique solutions which is more general.

Theorem 6 (Invariance Theorem) Let the assumption of Theorem 2. Suppose that there exists a function $V \in C^{1}\left(\mathbb{R}^{n} ; \mathbb{R}\right)$ such that $\dot{V}^{*} \leq 0$. Let $\Omega$ be a compact invariant subset of $\mathbb{R}^{n}, x_{0} \in \Omega$ and $x\left(\cdot ; x_{0}\right) \in \mathcal{S}\left(x_{0}\right)$ be a solution of $(6)$. Let $\mathcal{Z}=\left\{y \in \mathbb{R}^{n}: 0 \in \dot{V}(y)\right\}$ and $\mathcal{M}$ be the largest weakly invariant subset in the closure of $\mathcal{Z}$ then:

$$
\lim _{t \rightarrow+\infty} \operatorname{dist}\left(x\left(t ; x_{0}\right), \mathcal{M}\right)=0 .
$$

Proof Since $x_{0} \in \Omega$, and $\Omega$ is invariant, we have $\gamma(x) \subset \Omega$. Therefore, $\gamma(x)$ is bounded and:

$$
\lim _{t \rightarrow+\infty} \operatorname{dist}\left(x\left(t ; x_{0}\right), \Lambda(x)\right)=0 .
$$

It is enough to prove that $\Lambda(x) \subset \overline{\mathcal{Z}}$ due to the weak invariance of $\Lambda(x)$. Note that the function $V(\cdot)$ is $C^{1}$, it is bounded on the compact set $\Omega$. From Remark 3.2, we imply that $V(x(\cdot))$ is decreasing on $\mathbb{R}^{+}$since $\dot{V}^{*} \leq 0$. Therefore, there exists a real number $k$ such that $\lim _{t \rightarrow+\infty} V\left(x\left(t ; x_{0}\right)\right)=k$. For each $p \in \Lambda(x)$, there exist $\left\{t_{i}\right\}, t_{i} \rightarrow \infty$ as $i \rightarrow \infty$ and $x\left(t_{i} ; x_{0}\right) \rightarrow p$. Then, $V(p)=k$ due to the continuity of $V(\cdot)$. Hence, $V(p)=k$ for 
all $p \in \Lambda(x)$. Let $z \in \Lambda(x)$. Since $\Lambda(x)$ is weakly invariant, there exists a solution $\phi(t ; z)$ of (6) lying in $\Lambda(x)$. Therefore:

$$
V(\phi(t ; z))=k
$$

for all $t \geq 0$ which implies:

$$
0=\frac{d}{d t} V(\phi(t ; z)) \in \dot{V}(\phi(t ; z))
$$

for almost all $t \geq 0$. Hence, we have:

$$
\phi(t ; z) \in \mathcal{Z}
$$

for almost all $t \geq 0$. Since $\phi(\cdot ; z)$ is continuous, we obtain:

$$
z=\phi(0 ; z) \in \overline{\mathcal{Z}}
$$

and the result follows.

\section{Remark 7}

(1) The theorem is still true if we replace $\mathcal{Z}$ by $\mathcal{Z}_{\Omega}=\{y \in \Omega: 0 \in \dot{V}(y)\}$. It is enough to check that $\Lambda(x) \subset \overline{\mathcal{Z}}_{\Omega}$. Indeed, since $\underline{\gamma}(x) \subset \Omega$ and $\Omega$ is compact, we have $\Lambda(x) \subset \Omega$. Therefore, $\Lambda(x) \subset \overline{\mathcal{Z}} \cap \Omega \subset \overline{\mathcal{Z}}_{\Omega}$.

(2) We can "ignore" the role of the compact invariant set $\Omega$, provided we know that $x\left(\cdot ; x_{0}\right)$ is bounded. Indeed, from the proof above, if we have $x\left(\cdot ; x_{0}\right)$ is bounded then $\lim _{t \rightarrow+\infty} \operatorname{dist}\left(x\left(t ; x_{0}\right), \Lambda(x)\right)=0$ and we still have $\Lambda(x) \subset \overline{\mathcal{Z}}$. Therefore:

$$
\lim _{t \rightarrow+\infty} \operatorname{dist}\left(x\left(t ; x_{0}\right), \mathcal{M}\right)=0 .
$$

(3) By using the Invariance Theorem, we can obtain a stronger result than the one in Theorem 5.

\section{Corollary 1 Let the assumption of Theorem 2 and Assumption 1.}

(i) Suppose that there exists a Lyapunov function $V$ for problem (6) such that $\dot{V}^{*}(x)<0$ for all $x \in \overline{\mathbb{B}}_{\sigma} \backslash\{0\}$ and $\dot{V}^{*}(0)=0$. Then, the trivial solution is asymptotic stable.

(ii) If we have $\dot{V}^{*}(x)<0$ for all $x \in \mathbb{R}^{n} \backslash\{0\}, \dot{V}^{*}(0)=0$ and $V$ is radially unbounded, then 0 is globally asymptotic stable.

Proof

(i) It is easy to show that $\mathcal{Z}=\{0\}$. We have known that the trivial solution is stable. Hence, there exists $\delta>0$ such that for all $x_{0} \in \mathbb{R}^{n}$ and $\left\|x_{0}\right\| \leq \delta$, we have $x\left(t ; x_{0}\right) \in \overline{\mathbb{B}}_{\sigma}$ for all $t \geq 0$, i.e. $x\left(\cdot ; x_{0}\right)$ is bounded. The asymptotical stability of the trivial solution follows by using the Remark 7.2.

(ii) Given $x_{0} \in \mathbb{R}^{n}$, set $\Omega:=\left\{x \in \mathbb{R}^{n}: V(x) \leq V\left(x_{0}\right)\right\}$, then $x_{0} \in \Omega$ and $\Omega$ is a compact subset of $\mathbb{R}^{n}$ since $V$ is radially unbounded. It is sufficient to prove that it is invariant with respect to (6). Indeed, let $z \in \Omega$ and $x(\cdot ; z)$ be a solution of (6) satisfying $x(0 ; z)=z$. Since the mapping $V(x(t ; z))$ is decreasing with respect to 
$t$, we have $V(x(t ; z)) \leq V(x(0 ; z))=V(z) \leq V\left(x_{0}\right)$ for all $t \geq 0$. Hence, $x(t ; z) \in \Omega$ for all $t \geq 0$. The result follows by the Invariance Theorem with $\mathcal{Z}=\{0\}$.

Corollary 2 Let the assumption of Theorem 2. Suppose that there exists a radially unbounded function $V \in C^{1}\left(\mathbb{R}^{n} ; \mathbb{R}\right)$ such that $\dot{V}^{*} \leq 0$. Let $\mathcal{Z}=\left\{y \in \mathbb{R}^{n}: 0 \in \dot{V}(y)\right\}$ and $\mathcal{M}$ be the largest weakly invariant subset in the closure of $\mathcal{Z}$. Then, for any $x_{0} \in \mathbb{R}^{n}$ and $x\left(\cdot ; x_{0}\right) \in \mathcal{S}\left(x_{0}\right)$ a solution of $(6)$, we have:

$$
\lim _{t \rightarrow+\infty} \operatorname{dist}\left(x\left(t ; x_{0}\right), \mathcal{M}\right)=0 .
$$

Proof For each $x_{0} \in \mathbb{R}^{n}$, set $\Omega:=\left\{x \in \mathbb{R}^{n}: V(x) \leq V\left(x_{0}\right)\right\}$, then $x_{0} \in \Omega$ and $\Omega$ is an invariant compact subset of $\mathbb{R}^{n}$ (see the proof of Proposition 1.ii). Hence, the conclusion follows by the Invariance Theorem.

Theorem 7 Consider the differential inclusion system (6) with $A \in \mathbb{R}^{n \times n}$. Suppose that $F$ is upper semi-continuous with non-empty convex, compact values satisfying the linear growth condition and $-F$ is locally hypo-monotone. Furthermore:

(i) there exist a symmetric positive definite matrix $P \in \mathbb{R}^{n \times n}$ and a matrix $K \in \mathbb{R}^{n \times l}$ for some integer $l>0$ such that: $P A+A^{T} P=-K K^{T}$ and $\mathcal{C}^{T}=P B$;

(ii) $\sup _{w \in F(x)}\langle x, w\rangle \leq 0$ for all $x \in \mathbb{R}^{n}$.

Then, for each $x_{0} \in \mathbb{R}^{n}$, there exists a unique solution $x\left(t ; x_{0}\right)$ of (6). Furthermore, let $V(y):=\frac{1}{2} y^{T} P y, y \in \mathbb{R}^{n} ; \mathcal{Z}=\left\{y \in \mathbb{R}^{n}: 0 \in \dot{V}(y)\right\}$ and $\mathcal{M}$ be the largest invariant subset of $\mathcal{Z}$. Then:

$$
\lim _{t \rightarrow+\infty} \operatorname{dist}\left(x\left(t ; x_{0}\right), \mathcal{M}\right)=0
$$

Proof It is obvious that all the assumptions of Theorem 2 and 3 are satisfied. Indeed, we can choose the matrix $R=\sqrt{P}$. Therefore, for each $x_{0} \in \mathbb{R}^{n}$, there exists a unique solution $x\left(t ; x_{0}\right)$ of $(6)$. Note that $V$ is radially unbounded and its upper orbital derivative is:

$$
\begin{aligned}
\dot{V}^{*}(x) & =\sup _{w \in F(\mathcal{C} x)}\langle P x, A x+B w\rangle=\langle x, P A x\rangle+\sup _{w \in F(\mathcal{C} x)}\left\langle B^{T} P x, w\right\rangle \\
& =\langle x, P A x\rangle+\sup _{w \in F(\mathcal{C} x)}\langle\mathcal{C} x, w\rangle .
\end{aligned}
$$

From (ii), we have $\sup _{w \in F(\mathcal{C} x)}\langle\mathcal{C} x, w\rangle$ is non-positive. On the other hand, the term $\langle x, P A x\rangle=\frac{1}{2}\left\langle x,\left(P A+A^{T} P\right) x\right\rangle=-\frac{1}{2}\left\langle x, K K^{T} x\right\rangle=-\frac{1}{2}\left\langle K^{T} x, K^{T} x\right\rangle \leq 0$. It implies that the upper orbital derivative of $V$ is non-positive. The conclusion follows by Corollary 2.

\section{Remark 8}

(1) If $l=m$, we can use the Kalman-Yakubovich-Popov Lemma ([9,10]) to obtain the existence of $P$ and $K$ satisfying the condition (i) in Theorem 7.

(2) The condition (ii) means $x$ is contained in the polar cone of $F(x)$ for all $x \in \mathbb{R}^{n}$. The geometrical meaning in scalar case is that the graph of $-F$ belongs to the first 


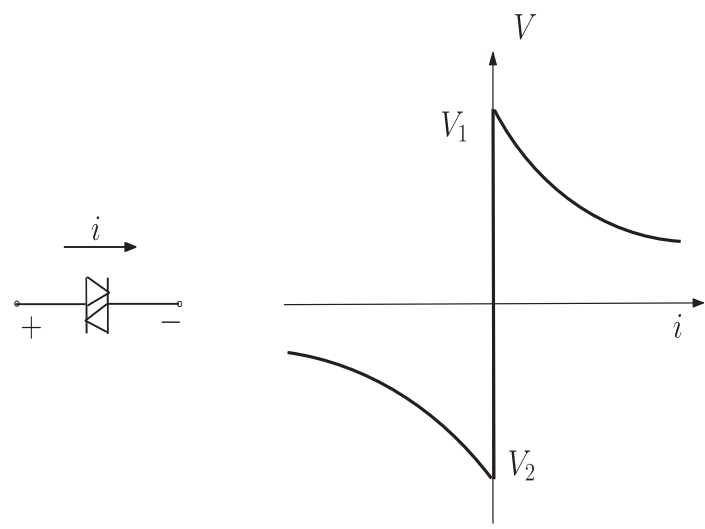

Figure 2. A typical voltage current characteristics of a diac.

and the third quadrants. It holds for a large class of function, for examples, when $-F$ is the characteristic function of a diode, Zener diode, diac, silicon controller rectifier etc. ([9]).

(3) If $K^{T}$ is full column rank, then $K K^{T}$ is positive definite. Then, it is easy to check that $\mathcal{Z}=\{0\}$. Therefore:

$$
\lim _{t \rightarrow+\infty} x\left(t ; x_{0}\right)=0
$$

Corollary 3 Consider the differential inclusion system (3) with $A \in \mathbb{R}^{n \times n}$ and $\mathcal{F}_{i}$ is upper semi-continuous with non-empty convex, compact values satisfying the linear growth condition and $-\mathcal{F}_{i}$ is locally hypo-monotone for $i=1,2, \ldots, m$. Suppose that:

(i) there exist a symmetric positive definite matrix $P \in \mathbb{R}^{n \times n}$ and a matrix $K \in \mathbb{R}^{n \times l}$ for some integer $l>0$ such that: $P A+A^{T} P=-K K^{T}$ and $\mathcal{C}^{T}=P B$;

(ii) $\sup _{w \in \mathcal{F}_{i}(y)}\langle y, w\rangle \leq 0$ for all $y \in \mathbb{R}, i=1,2, \ldots, m$.

Then, for each $x_{0} \in \mathbb{R}^{n}$, there exists a unique solution $x\left(t ; x_{0}\right)$ of (3). Furthermore, let $V(y):=\frac{1}{2} y^{T} P y, y \in \mathbb{R}^{n} ; \mathcal{Z}=\left\{y \in \mathbb{R}^{n}: 0 \in \dot{V}(y)\right\}$ and $\mathcal{M}$ be the largest invariant subset of $\mathcal{Z}$. Then:

$$
\lim _{t \rightarrow+\infty} \operatorname{dist}\left(x\left(t ; x_{0}\right), \mathcal{M}\right)=0 .
$$

Proof Let $F: \mathbb{R}^{m} \rightrightarrows \mathbb{R}^{m}$ defined in (4) and using Theorem 7 .

\section{Some examples in electronics}

Ampere-volt characteristics of some electrical devices: Electrical devices can be illustrated by their corresponding ampere-volt characteristics. Each device may possess various mathematical models based on the experimental measures. Figures 2 and 3 present the characteristics of a diac and a silicon controller rectifier which will be used in some circuit examples later (for reference about the ampere-volt characteristics of electrical devices, see [9]). 

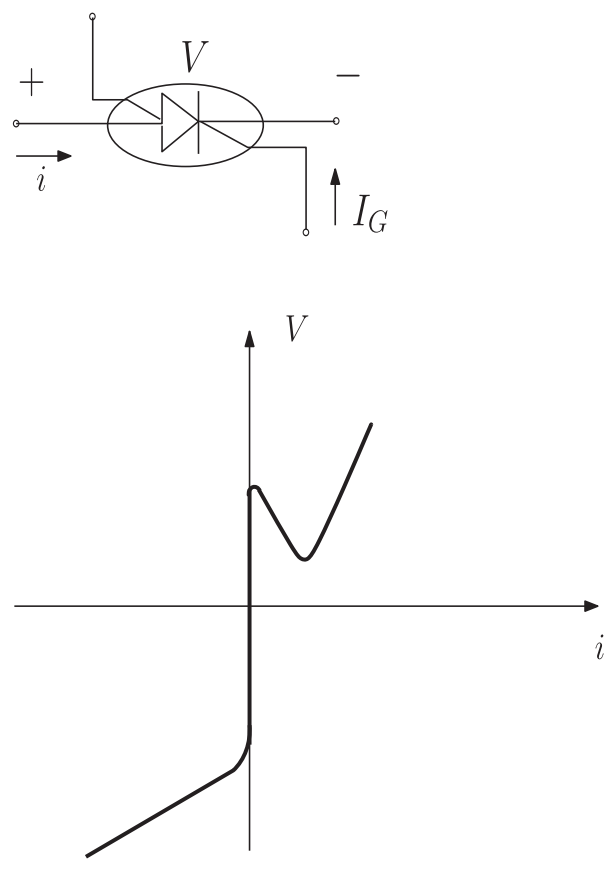

Figure 3. Silicon controller rectifier.

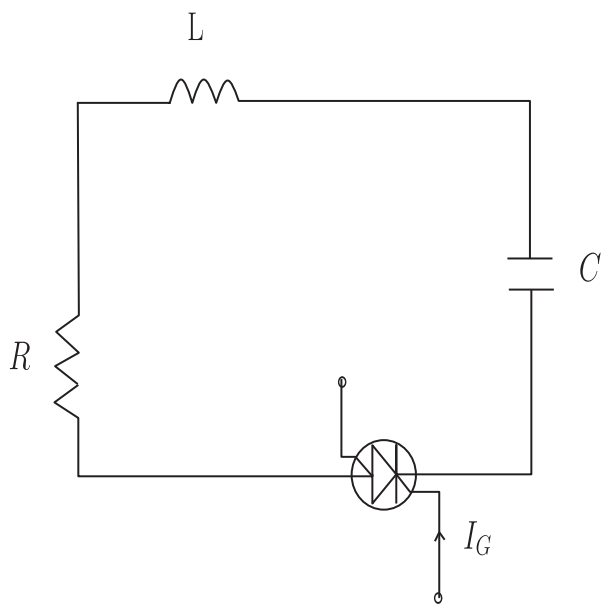

Figure 4. Circuit with silicon controller rectifier.

In both cases, the mappings ( $f_{\text {diac }}$ and $f_{\text {scr }}$ ) are set-valued where the values at 0 are intervals in $\mathbb{R}$ and single-valued differentiable with locally bounded derivatives in $\mathbb{R} \backslash\{0\}$. Hence, it can be checked that $f_{\text {diac }}$ and $f_{\text {scr }}$ are locally hypo-monotone. Furthermore, they are upper semi-continuous (their graphs are closed and locally bounded) with non-empty convex, compact values. They also have a property that for all $x \in \mathbb{R}$, the mappings $x f_{\text {diac }}(-x)$ and $x f_{\text {scr }}(-x)$ are single-valued and non-positive. 
Example 1 Consider the circuit in Figure 4 with a resistor $R>0$, an inductor $L>0$, a capacitor $C>0$. Let $x_{1}$ be the time integral of the current across the capacitance, $x_{2}$ the current across the circuit, $y_{L}$ is the voltage of the silicon controller rectifier. Using Kirchhoff's circuit laws, we have:

$$
\left(\begin{array}{c}
\dot{x}_{1} \\
\dot{x}_{2}
\end{array}\right)=\overbrace{\left(\begin{array}{cc}
0 & 1 \\
-\frac{1}{L C} & -\frac{R}{L}
\end{array}\right)}^{A}\left(\begin{array}{c}
x_{1} \\
x_{2}
\end{array}\right)-\overbrace{\left(\begin{array}{c}
0 \\
-\frac{1}{L}
\end{array}\right)}^{B} y_{L},
$$

where

$$
y=\overbrace{\left(\begin{array}{ll}
0-1
\end{array}\right)}^{\mathcal{C}}\left(\begin{array}{c}
x_{1} \\
x_{2}
\end{array}\right) \text { and } y_{L} \in f_{\mathrm{scr}}(y)=f_{\mathrm{scr}}\left(-x_{2}\right) .
$$

Let $\mathcal{Q}: \mathbb{R}^{2} \rightrightarrows \mathbb{R}^{2}, \mathcal{Q}(x)=A x-B f_{\text {scr }}(\mathcal{C} x)$ and $F:=-f_{\text {scr. }}$ The mapping $\mathcal{Q}$ is upper semi-continuous with non-empty convex compact values and the mapping $-F=f_{\text {scr }}$ is locally hypo-monotone. The matrix $R$ defined by:

$$
R=\left(\begin{array}{cc}
0 & 0 \\
0 & \sqrt{L}
\end{array}\right)
$$

holds $\mathcal{C}=B^{T} R^{T} R$. Therefore, the assumptions of Theorems 2, 3 and 4 are satisfied. Let $V(x)=\frac{\alpha_{1}}{2} x_{1}^{2}+\frac{\alpha_{2}}{2} x_{2}^{2}$ where $\alpha_{1}, \alpha_{2}>0$ can be chosen later, then $V$ is $C^{1}$, radially unbounded and:

$$
V^{\prime}(x)=\left(\begin{array}{c}
\alpha_{1} x_{1} \\
\alpha_{2} x_{2}
\end{array}\right)
$$

We have:

$$
\dot{V}(x)=\alpha_{1} x_{1} x_{2}+\alpha_{2} x_{2}\left(-\frac{1}{L C} x_{1}-\frac{R}{L} x_{2}+\frac{1}{L} f_{\text {scr }}\left(-x_{2}\right)\right)
$$

is single-valued since $x_{2} f_{\mathrm{scr}}\left(-x_{2}\right)$ is single-valued. We choose $\alpha_{1}=\frac{\alpha_{2}}{L C}$, then we obtain that:

$$
\dot{V}^{*}(x)=\dot{V}_{*}(x)=-\frac{R \alpha_{2}}{L} x_{2}^{2}+\frac{\alpha_{2}}{L} x_{2} f_{\text {scr }}\left(-x_{2}\right) \leq 0 .
$$

It is easy to check that $\mathcal{Z}=\left\{y \in \mathbb{R}^{2}: 0 \in \dot{V}(y)\right\}=\mathbb{R} \times\{0\}$ and the set of stationary solutions of the system $\mathcal{W}=\left\{\left(x_{1}, 0\right): x_{1} \in \mathbb{R}, x_{1} \in C f_{\text {scr }}(0)\right\}=C f_{\text {scr }}(0) \times\{0\}$. We can prove that $\mathcal{W}$ is the largest invariance subset of $\mathcal{Z}$. Indeed, let $\mathcal{D}$ be an invariance subset of $\mathcal{Z}$ and $z=\left(z_{1} z_{2}\right)^{T} \in \mathcal{D}$. The unique solution $\psi(\cdot ; z)=\left(\psi_{1}(\cdot ; z) \psi_{2}(\cdot ; z)\right)^{T}$ of $(12)$ satisfies $\psi(t ; z) \in \mathcal{D}$ for all $t \geq 0$. Then, for all $t \geq 0$, we have $\dot{\psi}_{1}(t ; z)=\psi_{2}(t ; z)=0$ which implies $\psi_{1}(t ; z) \equiv z_{1}, z_{2}=0$ and $z_{1} \in C f_{\text {scr }}(0)$. Therefore, we have: $\mathcal{D} \subset \mathcal{Z}$. Using Corollary 2, we obtain:

$$
\lim _{t \rightarrow+\infty} \operatorname{dist}\left(x_{1}(t), C f_{\mathrm{scr}}(0)\right)=0 \text { and } \lim _{t \rightarrow+\infty} x_{2}(t)=0 .
$$



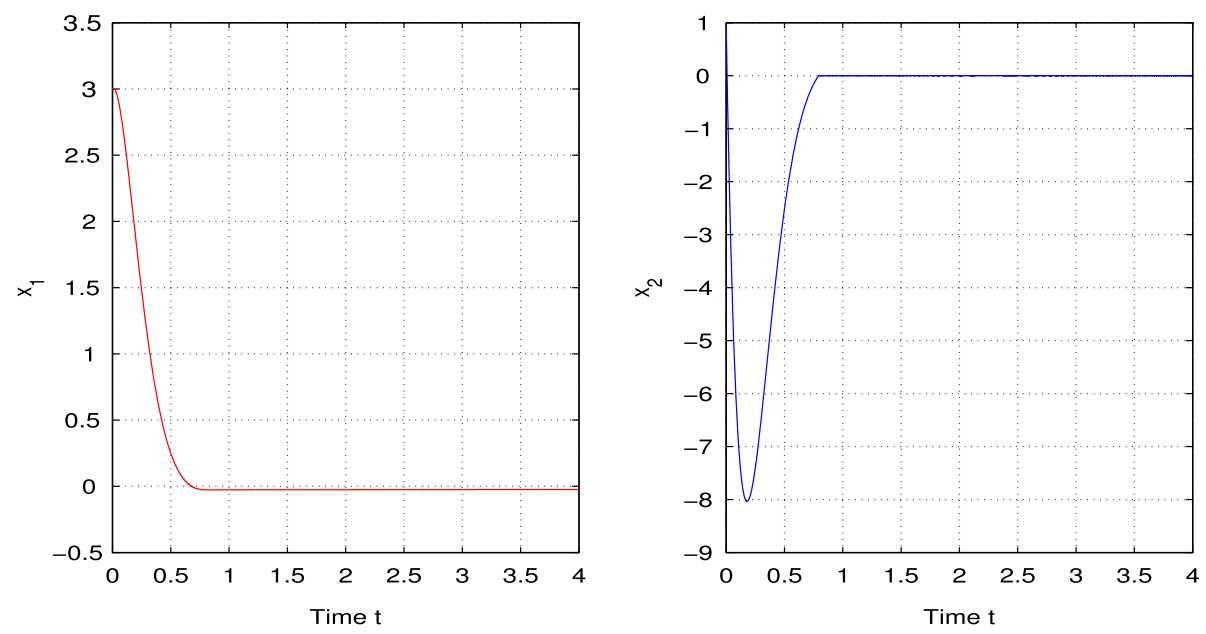

Figure 5. Numerical simulation for example 1. To view this figure in color, please see the online version.

Consider the circuit above which includes a voltage supply $u$. Then, the system becomes:

$$
\left(\begin{array}{c}
\dot{x}_{1} \\
\dot{x}_{2}
\end{array}\right)=\left(\begin{array}{cc}
0 & 1 \\
-\frac{1}{L C} & -\frac{R}{L}
\end{array}\right)\left(\begin{array}{c}
x_{1} \\
x_{2}
\end{array}\right)-\left(\begin{array}{c}
0 \\
-\frac{1}{L}
\end{array}\right) y_{L}+\left(\begin{array}{c}
0 \\
\frac{1}{L}
\end{array}\right) u,
$$

and

$$
\dot{V}^{*}(x)=\frac{d}{d t} V(x)=-\frac{R \alpha_{2}}{L} x_{2}^{2}+\frac{\alpha_{2}}{L} x_{2} y_{L}+\frac{\alpha_{2} u}{L} x_{2}
$$

Let $c:=\inf _{x \neq 0}\left|f_{\text {scr }}(x)\right|>0$. If $|u| \leq c$, we have $\frac{\alpha_{2}}{L} x_{2} y_{L}+\frac{\alpha_{2} u}{L} x_{2} \leq \frac{\alpha_{2}}{L}\left|x_{2}\right|\left(|u|-\left|y_{L}\right|\right) \leq 0$. Therefore, $\dot{V}^{*}(x) \leq 0$ and we obtain the stability of the trivial solution and the attractivity result as above. The set of stationary solutions then is $\mathcal{W}=\left\{\left(x_{1}, 0\right): x_{1} \in \mathbb{R}, x_{1} / C \in\right.$ $\left.f_{\text {scr }}(0)+u\right\}=C\left(f_{\text {scr }}(0)+u\right) \times\{0\}$ and we have:

$$
\lim _{t \rightarrow+\infty} \operatorname{dist}\left(x_{1}(t), C\left(f_{\mathrm{scr}}(0)+u\right)\right)=0 \text { and } \lim _{t \rightarrow+\infty} x_{2}(t)=0 .
$$

Note that we can use directly Theorem 7 with:

$$
P=\left(\begin{array}{cc}
1 / C & 0 \\
0 & L
\end{array}\right) \text { and } K=\left(\begin{array}{cc}
0 & 0 \\
0 & \sqrt{2 R}
\end{array}\right)
$$

which satisfy $P A+A^{T} P=-K K^{T}$ and $\mathcal{C}^{T}=P B$. The same analysis can be applied if we replace the function $f_{\text {scr }}$ by $f_{\text {diac }}$ (Figure 5). 


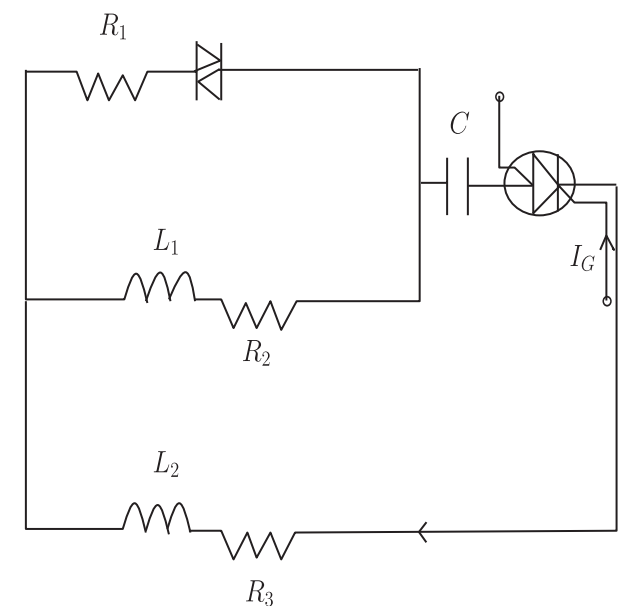

Figure 6. Circuit with silicon controller rectifier and diac.

Example 2 Next, we consider the circuit correspondent to the Figure 6. Applying Kirchhoff's circuit laws again, we obtain:

$$
\left(\begin{array}{c}
\dot{x}_{1} \\
\dot{x}_{2} \\
\dot{x}_{3}
\end{array}\right)=\overbrace{\left(\begin{array}{ccc}
0 & 1 & 0 \\
-\frac{1}{L_{2} C} & -\frac{R_{1}+R_{3}}{L_{2}} & \frac{R_{1}}{L_{2}} \\
0 & \frac{R_{1}}{L_{1}} & -\frac{R_{1}+R_{2}}{L_{1}}
\end{array}\right)}^{A}\left(\begin{array}{c}
x_{1} \\
x_{2} \\
x_{3}
\end{array}\right)-\overbrace{\left(\begin{array}{cc}
0 & 0 \\
\frac{1}{L_{2}} & \frac{1}{L_{2}} \\
-\frac{1}{L_{1}} & 0
\end{array}\right)}^{B}\left(\begin{array}{c}
y_{L_{1}} \\
y_{L_{2}}
\end{array}\right)
$$

and

$$
y_{L_{1}} \in f_{\text {diac }}\left(-x_{3}+x_{2}\right) \text { and } y_{L_{2}} \in f_{\text {scr }}\left(x_{2}\right),
$$

where $R_{1}, R_{2}, R_{3}>0$ are resistors, $L_{1}, L_{2}>0$ are inductors, $C>0$ is a capacitor, $x_{1}$ is the time integral of the current across the capacitor, $x_{2}$ is the current across the capacitor, $x_{3}$ is the current across the inductor $L_{1}, y_{L_{1}}$ is the voltage of the diac and $y_{L_{2}}$ is the voltage of the SCR. Let $\mathcal{C}:=\left(\begin{array}{ccc}0 & 1 & -1 \\ 0 & 1 & 0\end{array}\right)$ and $F: \mathbb{R}^{2} \rightrightarrows \mathbb{R}^{2}, x=\left(x_{1} x_{2}\right)^{T} \rightarrow-\left(\begin{array}{c}f_{\text {diac }}\left(x_{1}\right) \\ f_{\text {scr }}\left(x_{2}\right)\end{array}\right)$. Denote $\mathcal{Q}: \mathbb{R}^{3} \rightrightarrows \mathbb{R}^{3}$ the right-hand side of $(15)$, then for $x=\left(\begin{array}{lll}x_{1} & x_{2} & x_{3}\end{array}\right)^{T}$ we have:

$$
\mathcal{Q}(x)=A x+B F(\mathcal{C} x) .
$$

It is clear that $\mathcal{Q}$ is upper semi-continuous with non-empty convex compact values and the mapping $-F$ is locally hypo-monotone. The following matrix:

$$
R=\left(\begin{array}{ccc}
0 & 0 & 0 \\
0 & \sqrt{L_{2}} & 0 \\
0 & 0 & \sqrt{L_{1}}
\end{array}\right)
$$


satisfies $\mathcal{C}=B^{T} R^{T} R$. Hence, the assumptions of Theorems 2, 3 and 4 hold. We also consider the Lyapunov function of the form:

$$
V(x)=\frac{\alpha_{1}}{2} x_{1}^{2}+\frac{\alpha_{2}}{2} x_{2}^{2}+\frac{\alpha_{3}}{2} x_{3}^{2},
$$

where $\alpha_{1}, \alpha_{2}, \alpha_{3}>0$ can be chosen later. Let

$$
\begin{array}{cl}
\beta_{1}=\frac{1}{L_{2} C}, \quad \beta_{2}=\frac{R_{1}+R_{2}}{L_{2}}, & \beta_{3}=\frac{R_{1}}{L_{2}}, \quad \beta_{4}=\frac{1}{L_{2}}, \quad \beta_{5}=\frac{R_{1}}{L_{1}}, \\
\beta_{6}=\frac{R_{1}+R_{2}}{L_{1}}, \quad \beta_{7}=\frac{1}{L_{1}} .
\end{array}
$$

Then,

$$
\begin{aligned}
\dot{V}(x)= & \alpha_{1} x_{1} x_{2}+\alpha_{2} x_{2}\left(-\beta_{1} x_{1}-\beta_{2} x_{2}+\beta_{3} x_{3}-\beta_{4} f_{\text {diac }}\left(-x_{3}+x_{2}\right)-\beta_{4} f_{\text {scr }}\left(x_{2}\right)\right) \\
& +\alpha_{3} x_{3}\left(\beta_{5} x_{2}-\beta_{6} x_{3}+\beta_{7} f_{\text {diac }}\left(-x_{3}+x_{2}\right)\right) .
\end{aligned}
$$

We choose $\alpha_{1}, \alpha_{2}, \alpha_{3}$ such that $\alpha_{1}=\alpha_{2} \beta_{1}, \alpha_{2} \beta_{4}=\alpha_{3} \beta_{7}:=\gamma$ then we obtain:

$\dot{V}(x)=\left(\alpha_{2} \beta_{3}+\alpha_{3} \beta_{5}\right) x_{2} x_{3}-\alpha_{2} \beta_{2} x_{2}^{2}-\alpha_{3} \beta_{6} x_{3}^{2}+\gamma\left(x_{3}-x_{2}\right) f_{\text {diac }}\left(-x_{3}+x_{2}\right)-\alpha_{2} \beta_{4} x_{2} f_{\text {scr }}\left(x_{2}\right)$

is single valued since $\left(x_{3}-x_{2}\right) f_{\text {diac }}\left(-x_{3}+x_{2}\right)$ and $x_{2} f_{\text {scr }}\left(x_{2}\right)$ are single valued.

Replacing $\alpha_{3}=\alpha_{2} \frac{\beta_{4}}{\beta_{7}}$, we have that:

$$
\begin{aligned}
\alpha_{2} \beta_{3}+\alpha_{3} \beta_{5} & =\left(\beta_{3}+\frac{\beta_{4} \beta_{5}}{\beta_{7}}\right) \alpha_{2}=\frac{2 R_{1}}{L_{2}} \alpha_{2}, \quad \beta_{2}=\frac{R_{1}+R_{3}}{L_{2}}>\frac{R_{1}}{L_{2}}, \\
\alpha_{3} \beta_{6} & =\frac{\beta_{4} \beta_{6}}{\beta_{7}} \alpha_{2}=\frac{R_{1}+R_{2}}{L_{2}} \alpha_{2}>\frac{R_{1}}{L_{2}} \alpha_{2} .
\end{aligned}
$$

Therefore:

$$
\left(\alpha_{2} \beta_{3}+\alpha_{3} \beta_{5}\right) x_{2} x_{3}-\alpha_{2} \beta_{2} x_{2}^{2}-\alpha_{3} \beta_{6} x_{3}^{2} \leq \frac{R_{1}}{L_{2}} \alpha_{2}\left(2 x_{2} x_{3}-x_{2}^{2}-x_{3}^{2}\right)=-\frac{R_{1}}{L_{2}} \alpha_{2}\left(x_{2}-x_{3}\right)^{2} \leq 0 .
$$

Note that:

$$
\left(x_{3}-x_{2}\right) f_{\text {diac }}\left(-x_{3}+x_{2}\right) \leq 0 \text { and }-x_{2} f_{\text {scr }}\left(x_{2}\right) \leq 0 \text {. }
$$

So, we have:

$$
\dot{V}^{*}(x)=\dot{V}_{*}(x) \leq 0 .
$$

We can check that $\mathcal{Z}=\left\{y \in \mathbb{R}^{3}: 0 \in \dot{V}(y)\right\}=\mathbb{R} \times\{0\} \times\{0\}$. The set of stationary solutions $\mathcal{W}$ is a subset of $\mathcal{Z}$ and $\mathcal{W}=\left\{\left(x_{1}, 0,0\right): x_{1} \in \mathbb{R}, x_{1} \in-C\left(f_{\text {diac }}(0)+f_{\text {scr }}(0)\right\}=\right.$ $-C\left(f_{\text {diac }}(0)+f_{\text {scr }}(0)\right) \times\{0\} \times\{0\}$. Similarly, we can prove that $\mathcal{W}$ is the largest invariant subset of $\mathcal{Z}$ and by using Corollary 2, we have:

$\lim _{t \rightarrow+\infty} \operatorname{dist}\left(x_{1}(t),-C\left\{f_{\text {diac }}(0)+f_{\text {scr }}(0)\right\}\right)=0, \quad \lim _{t \rightarrow+\infty} x_{2}(t)=0$ and $\lim _{t \rightarrow+\infty} x_{3}(t)=0$.

We may also apply Theorem 7 for this circuit, which is left as an exercise for readers (Figure 7). 

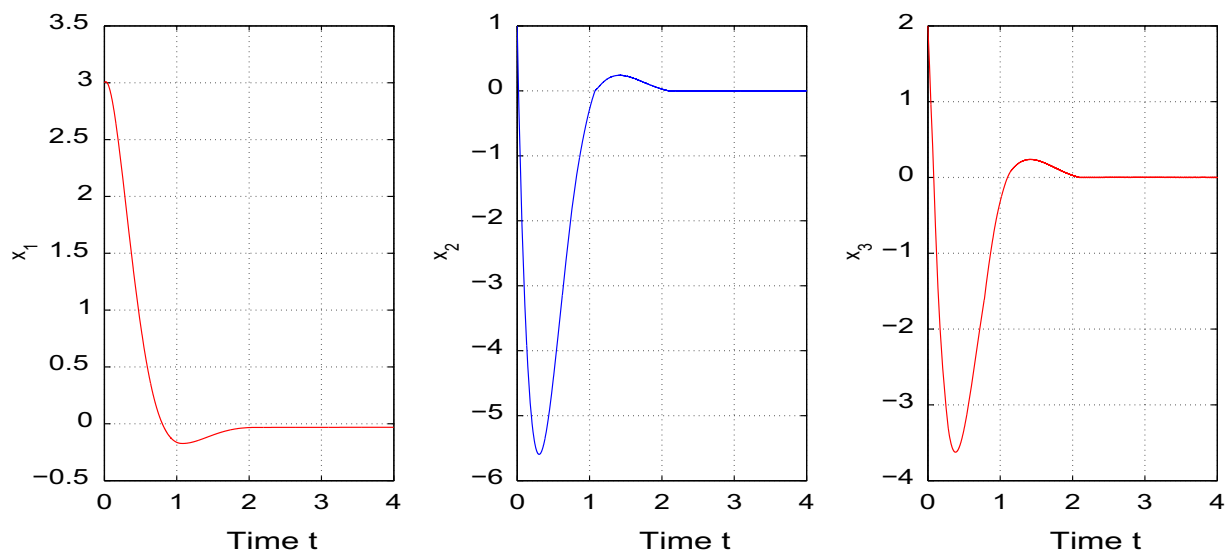

Figure 7. Numerical simulation for example 2. To view this figure in color, please see the online version.

\section{By way of conclusion}

In this paper, we have analysed the well-posedness of a class of non-motonone set-valued Lur'e dynamiacal systems. The existence and uniqueness of the trajectories are assured with a weaker assumption than the case of maximal monotone right-hand side. Some criterions for the Lyapunov function is also given to obtain the stability analysis and asymptotic properties of such systems. We developed an extended version of LaSalle's invariance principle which can be applied to study the attractivity of a stationary solution or a set. The case of $D \neq 0$ while the right-hand side is non-monotone is also interesting, but it is out of the scope of the current paper. It will be the subject of another paper in the future.

\section{References}

[1] Brogliato B, Daniliidis A, Lemaréchal C, Acary V. On the equivalence between complementarity systems, projected systems and differential inclusions. Systems and Control Letters. 2006;55(1):45-51.

[2] Dupuis P, Nagurney A. Dynamical systems and variational inequalities. Annals of Operations Research. 1993;44(1-4):9-42.

[3] Henry C. An existence theorem for a class of differential equations with multivalued right-hand side. Journal of Mathematical Analysis and Applications. 1973;41:179-186.

[4] Brogliato B, Goeleven D. Well-posedness, stability and invariance results for a class of multivalued Lur'e dynamical systems. Nonlinear Analysis: Theory, Methods and Applications. 2011;74:195-212.

[5] Aubin JP, Cellina A. Differential inclusions. Set-valued maps and viability theory. Berlin: Spinger-Verlag; 1984.

[6] Filippov AF. Differential equations with discontinuous right-hand side. Dordrecht: Kluwer Academic; 1988.

[7] Bacciotti A, Ceragioli F, Mazzi L. Differential inclusions and monotonicity conditions for nonsmooth Lyapunov functions. Set-Valued Analysis. 2000;8(3):299-309.

[8] Adly S, Brogliato B, Le BK. Well-posedness, robustness and stability analysis of a setvalued controller for Lagrangian systems. SIAM Journal on Control and Optimization. 2013;51(2):1592-1614. 
[9] Addi K, Adly S, Brogliato B, Goeleven D. A method using the approach of Moreau and Panagiotopoulos for the mathematical formulation of non-regular circuits in electronics. Nonlinear Analysis: Hybrid Systems. 2007;1(1):30-43.

[10] Brogliato B, Lozano R, Maschke B, Egeland O. Dissipative systems analysis and control. 2nd ed. London: Springer-Verlag; 2007.

[11] Adly S, Goeleven D. A stability theory for second-order nonsmooth dynamical systems with application to friction problems. Journal de Mathématiques Pures et Appliquées. 2004;83(1):1751.

[12] Adly S, Goeleven D, Le BK. Stability analysis and attractivity results of a DC-DC buck converter. Set-Valued and Variational Analysis. 2012;20:331-353.

[13] Mordukhovich BM. Variational analysis and generalized differentiation. I. Basic theory. Vol. 330, Grundlehren der Mathematischen Wissenschaften. Berlin: Springer-Verlag; 2006.

[14] Phelps RR. Convex functions, monotone operators and differentiability. Lecture Notes in Mathematics, Nr. 1364. Berlin: Springer-Verlag; 1989. Second Edition (1993).

[15] Rockafellar RT, Wets RJB. Variational analysis. Vol. 317, Grundlehren der Mathematischen Wissenschaften. Berlin: Springer; 1998.

[16] Rockafellar RT. Convex analysis. Princeton Mathematical Series, No. 28. Princeton, NJ: Princeton University Press; 1970. 